\title{
Application and research of loop-mediated isothermal amplification and lateral flow dipstick in detection of Porcine circovirus type 3
}

\section{Yan Wang}

Qingdao Agricultural University

\section{Xianjie Han}

Qingdao Agricultural University

Jianli Shi

Shandong Academy of Agricultural Sciences

Zhe Peng

Shandong Academy of Agricultural Sciences

Xiaoyan Wu

Shandong Academy of Agricultural Sciences

Shuo Wang

Shandong Normal University

\section{Panpan Sun}

Qingdao Agricultural University

Changxun Xin

Qingdao Agricultural University

Yuwei Liu

Shandong Normal University

\section{Shaojian Xu}

Shandong Academy of Agricultural Sciences Jun Li ( junli79@163.com)

\section{Research article}

Keywords: PCV3, LAMP-LFD, Sensitivity, Specificity, Convenience

Posted Date: September 27th, 2019

DOI: https://doi.org/10.21203/rs.2.15238/v1

License: (c) (1) This work is licensed under a Creative Commons Attribution 4.0 International License.

Read Full License 


\section{Abstract}

Background $\mathbb{B}$ Porcine circovirus type 3 (PCV3) is a newly detected pathogen from pigs in the past few years. As the infection rate of PCV3 in the herd is getting higher and wider, the infection is more and more serious. The virus with unclear clinical symptoms and pathogenesis is also paid more and more attention. And detection of pathogenic antibodies has become an important issue. Methods:In this experiment, Loop-mediated isothermal amplification (LAMP) was combined with Lateral flow dipstick (LFD) to establish a rapid and convenient detection method (LAMP-LFD). Results:The results showed that the band was darkened at a nucleic acid concentration of $0.2 \mathrm{pg} / \mu \mathrm{L}$ using a conventional PCR method, and a clear detection line was observed at a nucleic acid concentration of $0.2 \mathrm{fg} / \mu \mathrm{L}$ using the LAMP-LFD method. The primer and hybridization probe are only compatible with PCV3.Compared to the PCR method, the LAMP-LFD method has a shorter time (about $1 \mathrm{~h}$ ) and requires less instrumentation. Conclusions: The test results show that the LAMP-LFD method has extremely high sensitivity and specificity. The operation is simple and quick. The visual effect of the detection results is better than that of PCR and single LAMP test, which can bring convenience in practical production applications.

\section{Background}

Porcine circovirus (PCV) is the smallest virus known to infect mammals today, and a single-stranded circular DNA virus that is about $1.7 \mathrm{k} \mathrm{kb}$ in size. Porcine circovirus has been found to have three subtypes, PCV1, PCV2 and PCV3. Pigs infected with PCV1 do not have clinical symptoms(1); pigs infected with PCV2 can cause multiple clinically complicated disease such as weaned piglet multisystem exhaustion syndrome (PMWS), dermatitis nephropathy, progressive wasting, poor growth and development.PCV3 is a new subtype identified from pigs, and its clinical symptoms and pathogenesis are still unclear(2,3). In 2017, PCV3 was first detected and reported in southern China (4).

The infection rate of PCV3 has increased year by year and the infection range has become larger and larger. Although the clinical symptoms of PCV3 are not yet clear, PCV3 does cause tissue inflammation and has a high detection rate in sick pigs with symptoms of diarrhea, hyperthermia or respiratory diseases. Whether PCV3 is destructive to the body's immune system after invading the body needs to be known by studying its pathogenic mechanism. Due to the increasing number of positive poisoned pigs, the rapid detection of PCV3 has become one of the problems of various farming units.

Loop-mediated isothermal amplification (LAMP) is a nucleic acid in vitro amplification technique (5). DNA was amplified specifically, efficiently, and rapidly using four specially designed primers and Bst DNA polymerase with strand displacement activity under constant temperature conditions. LAMP technology has been widely used in the fields of nucleic acid scientific research, disease diagnosis and genetically modified food detection due to its strong specificity, high sensitivity, fastness, accuracy and ease of operation. Compared with PCR technology, LAMP technology greatly simplifies the requirements of the instrument, and the reaction time is greatly shortened, which is more suitable for fast and simple detection. 
The basis of the lateral flow dipstick (LFD) technology is the highly sensitive technique for detecting trace antigens established by Sano et al. in 1992(6). The LFD method mainly uses colloidal gold technology. Biotin is labeled on the primer, and the biotin-labeled amplification product can specifically hybridize with the probe labeled with fluorescein isothiocyanate (FITC) and combine with the antibody against FITC to form a ternary complex. This ternary complex is bound to the detection line containing biotin antibodies. The unhybridized FITC-labeled probes form a biotin-free binary complex that binds to the FITC-containing quality control line (7).When the quality control line and the test line appear on the test strip at the same time, the test sample is positive.

The LAMP product can be judged by agarose gel electrophoresis to observe the band, using a turbidimeter or using a fluorescent dye to observe the color change. These determination methods have some shortcomings. The LAMP-LFD technique presents the LAMP product in a color-developing manner on the lateral flow test strip, and the results can be judged by visual observation. This method sheds the dependence on the instrument, discards the toxic reagents, and because the introduction of specific probes makes the results more specific, and the entire detection time is only increased by $8-10$ min based on the LAMP reaction time.

This test uses the LAMP-LFD method to detect PCV3, and performs specificity, sensitivity and reproducibility tests to make the test results more accurate, and 100 clinical samples were detected using the LAMP-LFD method. It is designed to obtain test results at a higher speed, convenience and reliability in actual production and life.

\section{Methods}

\subsection{Virus and samples}

Porcine circovirus type 3(PCV3) nucleic acid and plasmid, porcine circovirus type 1/2(PCV1/2), porcine respiratory and reproductive system syndrome virus(PRRSV), swine fever virus(CSFV), and pseudorabies virus(PRV), etc. are all from the Shandong Provincial Key Laboratory of Prevention and Control of Livestock and Poultry Diseases. 100 samples used in clinical trials were from farms in Yantai, Linyi and Liaocheng.

\subsection{LAMP-related primers and hybrid probe design}

Refer to the PCV3 related conserved sequence uploaded by NCBI (GenBank accession number: MH107164.1), and design a set of LAMP using the LAMP primer online design website (http://primerexplorer.jp/elamp4.0.0/index.html). The primers include an upstream outer primer F3 (TCCAGTTTTTTCCGGGACAT), a downstream outer primer B3 (AACACTTGGCTCCAAGAC), an upstream inner primer FIP (CTTTTTCTCCAGACCCACCCCA-AAAGCAGTGCTCCCCATTG), and a downstream inner primer BIP (TTCCCGCCAGAATTGGTTTGG-GCGGAAAGTTCCACTCGTAA), as shown in Figure.1. A plurality of hybridization probes were designed using Primer 5.0 for screening according to the target amplified fragment, as shown in Table 1. The primers and hybridization probes were synthesized and 
modified by Bao Bio (Dalian) Co., Ltd. FIP 5' end modified biotin (Biotin), hybridization probe 5' end modified fluorescein isothiocyanate (FITC).

\subsection{Conventional PCR method}

The external primer F3/B3 was used as upstream and downstream primer for conventional PCR, and the PCR reaction system was prepared to be $20 \mu \mathrm{L}$ according to the instructions of Nanjing Vazyma Biotechnology Co., Ltd. 2×Taq Plus Master Mix II, as shown below:

\begin{tabular}{ll}
\hline 2×Taq Plus Master Mix $\searrow$ & $10 \mu \mathrm{L}$ \\
\hline F3 & $1 \mu \mathrm{L}$ \\
\hline B3 & $1 \mu \mathrm{L}$ \\
\hline DNA Sample & $1.5 \mu \mathrm{L}$ \\
\hline $\mathrm{ddH}_{2} \mathrm{O}$ & $6.5 \mu \mathrm{L}$
\end{tabular}

Reaction conditions: denaturation $94{ }^{\circ} \mathrm{C}, 4 \mathrm{~min} ; 94^{\circ} \mathrm{C} 30 \mathrm{~s}, 57^{\circ} \mathrm{C} 30 \mathrm{~s}, 72{ }^{\circ} \mathrm{C} 30 \mathrm{~s}$, a total of 32 cycles; total extension $72{ }^{\circ} \mathrm{C}, 8 \mathrm{~min}$. After the completion of the reaction, the product was stored at $4{ }^{\circ} \mathrm{C}$, subjected to $1.5 \%$ agarose gel electrophoresis, and observed under a gel imager.

\subsection{Establish LAMP system and condition optimization}

Using a PCV3 positive plasmid as a DNA template and $25 \mu \mathrm{L}$ of the LAMP system was prepared according to the NewEngland Biolabs Bst 2.0 DNA polymerase instructions as follows:

\begin{tabular}{|c|c|}
\hline 10x ThermoPol ${ }^{\circledR}$ Buffer & $2.5 \mu \mathrm{L}$ \\
\hline $\mathrm{MgSO}_{4} \otimes 100 \mathrm{mmol} / \mathrm{L} \nabla$ & $1.5 \mu \mathrm{L}$ \\
\hline dNTP Mix $\llbracket 2.5 \mathrm{mM} \otimes N T P$ rMO & $5 \mu \mathrm{L}$ \\
\hline Betaine & $2 \mu \mathrm{L}$ \\
\hline F3/B3囚10 mmol/L】 & $0.25 \mu \mathrm{L}$ \\
\hline $\mathrm{FIP} / \mathrm{BIP} \otimes 10 \mathrm{mmol} / \mathrm{L} \rrbracket$ & $1 \mu \mathrm{L}$ \\
\hline Bst DNA Polymerase $₫ 8000 \mathrm{U} / \mathrm{mL} \rrbracket$ & $1.5 \mu \mathrm{L}$ \\
\hline DNA Sample & $1.5 \mu \mathrm{L}$ \\
\hline Nuclease-free Water & add to $25 \mu \mathrm{L}$ \\
\hline
\end{tabular}


The optimal reaction time was explored in a $60^{\circ} \mathrm{C}$ water bath. The reaction time was set at $35,40,45,50$, $55,60,65,70$, and 75 minutes for each gradient. After the LAMP reaction time was selected, the optimal reaction temperature exploration test was carried out, and the reaction temperatures of $56,58,59,60,61$, 62,63 , and $64^{\circ} \mathrm{C}$ were respectively set. Determine the optimal reaction time and temperature, in order to obtain the experimental results quickly and easily in the actual application process.

\subsection{LAMP-LFD detection}

The test strips used in the LFD test were from Milenia ${ }^{\circledR}$ HybriDetect 2T. After the LAMP reaction was completed, $2 \mu \mathrm{L}$ of hybridization probe was added to continue the reaction for 5- $6 \mathrm{~min}$, and then $5 \mu \mathrm{L}$ of the product was taken and added to $100 \mu \mathrm{L}$ of HybriDetect Assay Buffer for 1-2 min. The test results were observed after adding a lateral flow test strip.

\subsection{Specificity and sensitivity test of LAMP-LFD detection}

Under optimized conditions, the LAMP-LFD specificity tests were performed simultaneously using PCV1, PCV2, PRV, CSFV, PRRSV and PCV3 as templates. Under optimized conditions, the PCV3 positive plasmid was diluted 10-fold (initial concentration was $200 \mathrm{ng} / \mu \mathrm{L}$ ) for routine PCR and LAMP-LFD sensitivity tests.

\subsection{Repeatability testing and clinical application of LAMP-LFD detection}

Under optimized conditions, the LAMP-LFD repeatability test was carried out using a PCV3 positive plasmid (plasmid concentration: $200 \mathrm{ng} / \mu \mathrm{L}$ and the lowest concentration in the sensitivity test, respectively) as a template.

The conventional PCR and LAMP-LFD methods were used to detect 100 suspected diseased pig tissues from Yantai, Linyi and Liaocheng in Shandong Province to evaluate the applicability of the LAMP-LFD method in clinical testing.

\section{Results}

\subsection{LAMP-LFD detection and hybridization probe selection}

The optimal reaction time temperature of PCV3 LAMP was 50 min and $62{ }^{\circ} \mathrm{C}$ (shown in Figure.2). The optimal reaction conditions were used to select hybridization probes. The results showed that the hybridization effect of Probe 1 and Probe A was better than others (shown in Figure.3). Finally, Probe 1 was selected.

\subsection{Specificity and sensitivity test}

The LMAP primer only amplifies PCV3 and does not react with other viral nucleic acids, demonstrating its specificity (Figure 4 and 5). Sensitivity tests showed that the conventional PCR method was obscured when the nucleic acid dilution gradient was 10-6 (this gradient concentration was $0.2 \mathrm{pg} / \mu \mathrm{L}$, shown in 
Figure 6), while the LAMP-LFD method still observed a clear detection line at a dilution gradient of 109 (this gradient concentration is $0.2 \mathrm{fg} / \mu \mathrm{L}$, shown in Figure 7). It shows that the LAMP-LFD detection system is nearly 1000 times more sensitive than the conventional PCR method.

\subsection{Repeatability test and clinical application of LAMP-LFD method}

The results of the reproducibility test showed that clear detection lines and quality control lines were observed in the other four tests except that the detection line of the lower component of the third test plasmid was unclear (shown in Figure 8). It shows that LAMP-LFD detection technology can achieve high reproducibility even at low nucleic acid concentrations.

100 samples were subjected to PCV3 detection by conventional PCR and LAMP-LFD methods. The detection rate of PCV3 using conventional PCR method was 83\% (83/100) (shown in Figure 9, results of partial sample), while the detection rate of PCV3 using the LAMP-LFD method was 85\% (85/100)(shown in Figure 10, results of partial sample). Furthermore, all 83 positive samples detected by the LAMP method were included in 85 positive samples detected by the LAMP-LFD method.

\section{Discussion}

As a newly discovered virus subtype in recent years, PCV3 has not been found to bring obvious clinical symptoms to infected pigs due to the lack of mature technology for isolation and culture. However, there are reports that the incidence of PCV3 is higher in pigs with severe diarrhea and respiratory diseases. Since the discovery and reporting of PCV3 by Rachel Palinski et al in 2016(3), countries have detected and reported them. In the first report of the discovery of PCV3-positive pigs in southern China in 2017(4), the presence of PCV3 was first discovered in Shandong Province $(8,9)$. Although no corresponding clinical symptoms were found in infected pigs, an inflammatory response was observed from the tissue sections. The detection of antibodies by Enzyme linked immunosorbent assay (ELISA) (10) and the detection of antigen by PCR (11) have found that the positive rate of PCV3 in pigs in China is high. This shows that the situation of pigs infected with PCV3 is very serious and the scope is getting wider and wider, which must be taken seriously.

As a simple and fast method for nucleic acid amplification, LAMP has been used more frequently in recent years. However, since the visualization effect of the LAMP results is too complicated compared to the LAMP operation itself, for example, the use of agarose gel electrophoresis or a turbidimeter makes many people, especially non-scientific personnel, less enthusiastic about LAMP technology. The LAMPLFD method can just improve the visualization of the test results, and the operation is simple and convenient. Now, there are reports that the LAMP-LFD technology is used to detect swine fever virus and various bacteria (12-14). The results of this experiment show that the sensitivity of the LAMP-LFD method is much higher than that of conventional PCR in terms of sensitivity, and the reproducibility of the LAMP-LFD method is highly reproducible for clinical rapid detection. In the clinical test experiment, the detection rate was slightly higher by the LAMP-LFD method than the conventional PCR method. At the same time, the LAMP-LFD method also detected positive samples that were not detected using 
conventional PCR. Combined with the high sensitivity of the LAMP-LFD method, the LAMP-LFD method is more advantageous in clinical testing. Moreover, the LAMP-LFD method does not require exposure to toxic reagents, nor expensive instruments, and the time for obtaining results is short. These advantages will bring great convenience to the farming unit in practical production applications.

\section{Conclusions}

The test results show that the LAMP-LFD method has extremely high sensitivity and specificity. The operation is simple and quick. The visual effect of the detection results is better than that of PCR and single LAMP test, which can bring convenience in practical production applications. The experimental results show that the LAMP-LFD method has more excellent effects in terms of specificity and sensitivity than the conventional PCR method. Moreover, the visualization of the LAMP-LFD method is more clear. Compared with the conventional PCR method and the LAMP method, the LAMP-LFD method has the advantages of high efficiency and convenience, and the detection result can be quickly obtained in actual production applications.

\section{Abbreviations}

Abbrevation Words Table

\begin{tabular}{ll} 
ABBREVATION & FULL NAME \\
PCV1/PCV2/PCV3 & Porcine circovirus type 1/2/3 \\
\hline PMWS & Piglet multisystem exhaustion syndrome \\
\hline LAMP & Loop-mediated isothermal amplification \\
LFD & Lateral flow dipstick \\
FITC & Fluorescein isothiocyanate \\
PRRSV & Porcine respiratory and reproductive system syndrome virus \\
CSFV & Cwine fever virus \\
PRV & Pseudorabies virus \\
ELISA & Enzyme linked immunosorbent assay
\end{tabular}

\section{Declarations}

Ethics approval and consent to participate

Not applicable

Consent to publication 
All authors contributed to manuscript revision, read and approved the submitted version. All authors agree that the manuscript is published.

\section{Availability of data and material}

All data are fully available without restriction.

\section{Competing interests}

The authors declare that the research was conducted in the absence of any commercial or financial relationships.

\section{Funding}

The study was partly supported by National Key R\&DProgram (2016YFD0500708), Shandong Province Modern Agricultural Industry Technology System (SDAIT-08-07), Doctor Fund of Shandong Province (ZR2017BC087), Shandong natural fund (ZR2017YL015, ZR2017YL014), Taishan Scholars Project, Agricultural Science and Technology Innovation Project of Shandong Academy of Agricultural Sciences (CXGC2016B14).

\section{Authors' contributions}

YAW, XJ, JUL contributed to the concept and design of the research; JIL, ZP, XY, PP, CX, SW, YUW contributed to the experimental research process; YAW wrote the first draft of the manuscript; $X \mathrm{~J}$, JUL wrote sections of the manuscript ;YAW, JIL, ZP, XY, SJ, JUL analyzed and discussed the research results.

\section{Acknowledgements}

Thanks to myself for insisting on not giving up during the study to complete the study. I sincerely thank my tutor and my classmates for helping me in all aspects. Thanks to the collaborators, with their help, can successfully complete the research and writing of the article. Thanks to the Shandong Provincial Key Laboratory of Animal Disease Control \& Breeding for giving me a good atmosphere for research.

\section{References}

1. S. Krakowka, J. A. Ellis, B. Meehan, S. Kennedy, F. McNeilly and G. Allan. 2000. Viral Wasting Syndrome of Swine: Experimental Reproduction of Postweaning Multisystemic Wasting Syndrome in Gnotobiotic Swine by Coinfection with Porcine Circovirus 2 and Porcine Parvovirus. Veterinary Pathology 37(3). 254-263.

2. Tung Gia Phan , Federico Giannitti , Stephanie Rossow , Douglas Marthaler , Todd Knutson , Linlin Li ,Xutao Deng , Talita Resende , Fabio Vannucci and Eric Delwart. 2016. Detection of a novel circovirus PCV3 in pigs with cardiac and multi-systemic inflammation. Virology Journal. 10.1186/s12985-016-0642-z. 
3. Palinski Rachel, Piñeyro Pablo, Shang Pengcheng ,Yuan Fangfeng , Guo Rui ,Fang Ying,Byers Emily,Hause Ben M. 2017. A Novel Porcine Circovirus Distantly Related to Known Circoviruses Is Associated with Porcine Dermatitis and Nephropathy Syndrome and Reproductive Failure. Journal of virology 91(1).

4. Shen H, Liu X , Zhang P , Wang L , Liu Y ,Zhang L ,Liang P ,Song C. 2018. Genome characterization of a porcine circovirus type 3 in South China. Transboundary and Emerging Diseases 65(1). 264-266.

5. Tsugunori Notomi, Hiroto Okayama, Harumi Masubuchi, Toshihiro Yonekawa ,Keiko Watanabe , Nobuyuki Amino and Tetsu Hase. 2000. Loop-mediated isothermal amplification of DNA. Nucleic Acids Research 28(12).

6. T. Sano, C. L. SmithandC. R. Cantor. 1992. Immuno-PCR: very sensitive antigen detection by means of specific antibody-DNA conjugates. Science 258(5079). 120-2. 10.1126/science.1439758.

7. Glynou K, loannou PC, Christopoulos TK and Syriopoulou V. 2003. Oligonucleotide-functionalized gold nanoparticles as probes in a dry-reagent strip biosensor for DNA analysis by hybridization. Anal Chem 75(16). 4155-4160.

8. S. Zheng,J. Shi,X. Wu,Z. Peng,C. Xin,L. Zhang,Y. Liu,M. Gao,S. Xu,H. Han,J. Yu,W. Sun,X. Cong,J. LiandJ. Wang. 2018. Presence of Torque teno sus virus 1 and 2 in porcine circovirus 3-positive pigs. Transbound Emerg Dis 65(2). 327-330.

9. S. Zheng,X. Wu,L. Zhang,C. Xin,Y. Liu,J. Shi,Z. Peng,S. Xu,F. Fu,J. Yu,W. Sun,S. Xu,J. LiandJ. Wang. 2017. The occurrence of porcine circovirus 3 without clinical infection signs in Shandong province. Transbound Emerg Dis 641337-1341.

10. Junhua Deng,Xiangdong Li,DingDing Zheng,Yanwei Wang,Lingyan Chen,Huanhuan Song,Tongyan Wang,Yuxin Huang,Wenqiang PangandKegong Tian. 2018. Establishment and application of an indirect ELISA for porcine circovirus 3. Archives of Virology 163(2). 479-482.

11. Shao-Lun Zhai,Xia Zhou,He Zhang,Ben M. Hause,Tao Lin,Runxia Liu,Qin-Ling Chen,Wen-Kang Wei,Dian-Hong Lv,Xiao-Hui Wen,Feng LiandDan Wang. 2017. Comparative epidemiology of porcine circovirus type 3 in pigs with different clinical presentations. Virology journal 14(1). 222-6.

12. Junling ZHU, Zuodong YE, Jieru DENG, Hongchao GOU, Jinding CHEN. 2016. Rapid detection of classical swine fever virus by loop-mediatedisothermal amplification combined with lateral flow dipstick method. Journal of South China Agricultural University 37(1). 1-7.

13. Marco Lalle, Alessia Possenti, Jitender P. Dubey and Edoardo Pozio. 2018. Loop-Mediated Isothermal Amplification-Lateral-Flow Dipstick (LAMP-LFD) to detect Toxoplasma gondii oocyst in ready-to-eat salad. Food Microbiology (70)137-142.

14. Darin Kongkasuriyachai, Suganya Yongkiettrakul, Wansika Kiatpathomchai and Narong Arunrut. 2017. Loop-Mediated Isothermal Amplification and LFD Combinationfor Detection of Plasmodium falciparum and Plasmodium vivax. Methods in molecular biology (Clifton, N.J.). 431-443.

\section{Tables}


Table 1 Hybrid probe

\begin{tabular}{|c|c|c|}
\hline Name & Sequence $45 \square-3 \square \square$ & Length $\square b p \square$ \\
\hline Probe 1 & CTTGTCCTGGGTGAGC & 17 \\
\hline Probe 2 & TTGTCCTGGGTGAGCG & 16 \\
\hline Probe 3 & AGAAGAGGCTTTGTCC & 16 \\
\hline Probe A & GGGGTGAAGTAACGG & 15 \\
\hline Probe B & TCCGCATAAGGGTC & 14 \\
\hline
\end{tabular}

\section{Figures}

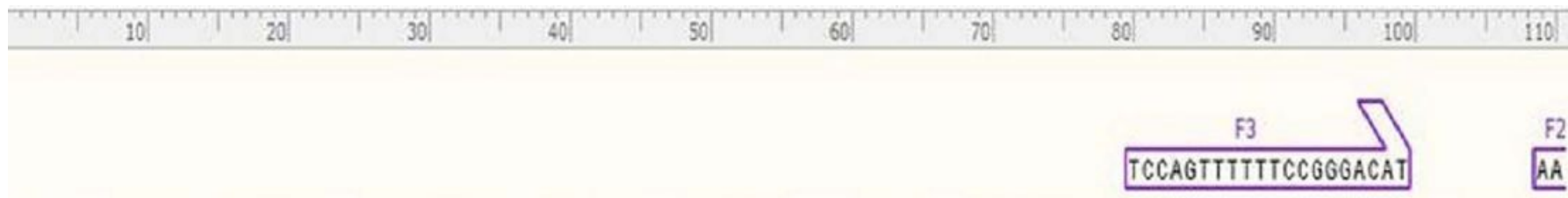

TCAGATTTATATTTATTTTCAGTTAGAGAACGGACTTGTAACGAATCCAAACTTCTTTGGTGCCGTAGAAGTCTGTCATTCCAGTTTTTTCCGGGACATAAATGCTCCAA

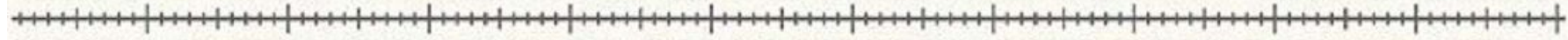
AGTCTAAATATAAATAAAAGTCAATCTCTTGCCTGAACATTGCTTAGGTTTGAAGAAACCACGGCATCTTCAGACAGTAAGGTCAAAAAAGGCCCTGTATTTACGAGGTT

$\frac{\text { F2 }}{\text { AGCAGTGCTCCCCATTG }}$

BTCCCGCCAGAT AGCAGTGCTCCCCATTGAACGGTGGGGTCATATGTGTTGAGCCATGGGGTGGGTCTGGAGAAAAAGAAGAGGCTTTGTCCTGGGTGAGCGCTGGTAGTTCCCGCCAGAAT

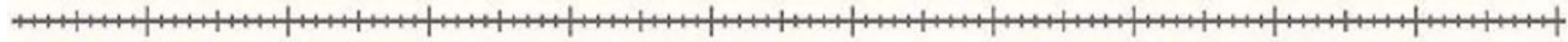
TCGTCACGAGGGGTAACTTGCCACCCCAGTATACACAACTCGGTACCCCACCCAGACCTCTTITTCTTCTCCGAAACAGGACCCACTCGCGACCATCAAGGGCGGTCTTA

$\sqrt{\text { ACCCCACCCAGACCTCTTTTTC }}$

TOGTTTGO

TGGTTTGGGGGTGAAGTAACGGCTGTGTITTTIITTAGAAGTCATAACTITACGAGTGGAACTTTCCGCATAAGGGTAGTCTTGGAGCCAAGTGTTTGTGGTCCAGGCGC HOH ACCAAACCCCCACTTCATTGCCGACACAAAAAAAAATCTTCAGTATTGAAATGCTCACCTTGAAAGGCGTATTCCCATCAGAACCTCGGTTCACAAACACCAGGTCCGCG

AATGCTCACCTTGAAAGGCG

\section{Figure 1}



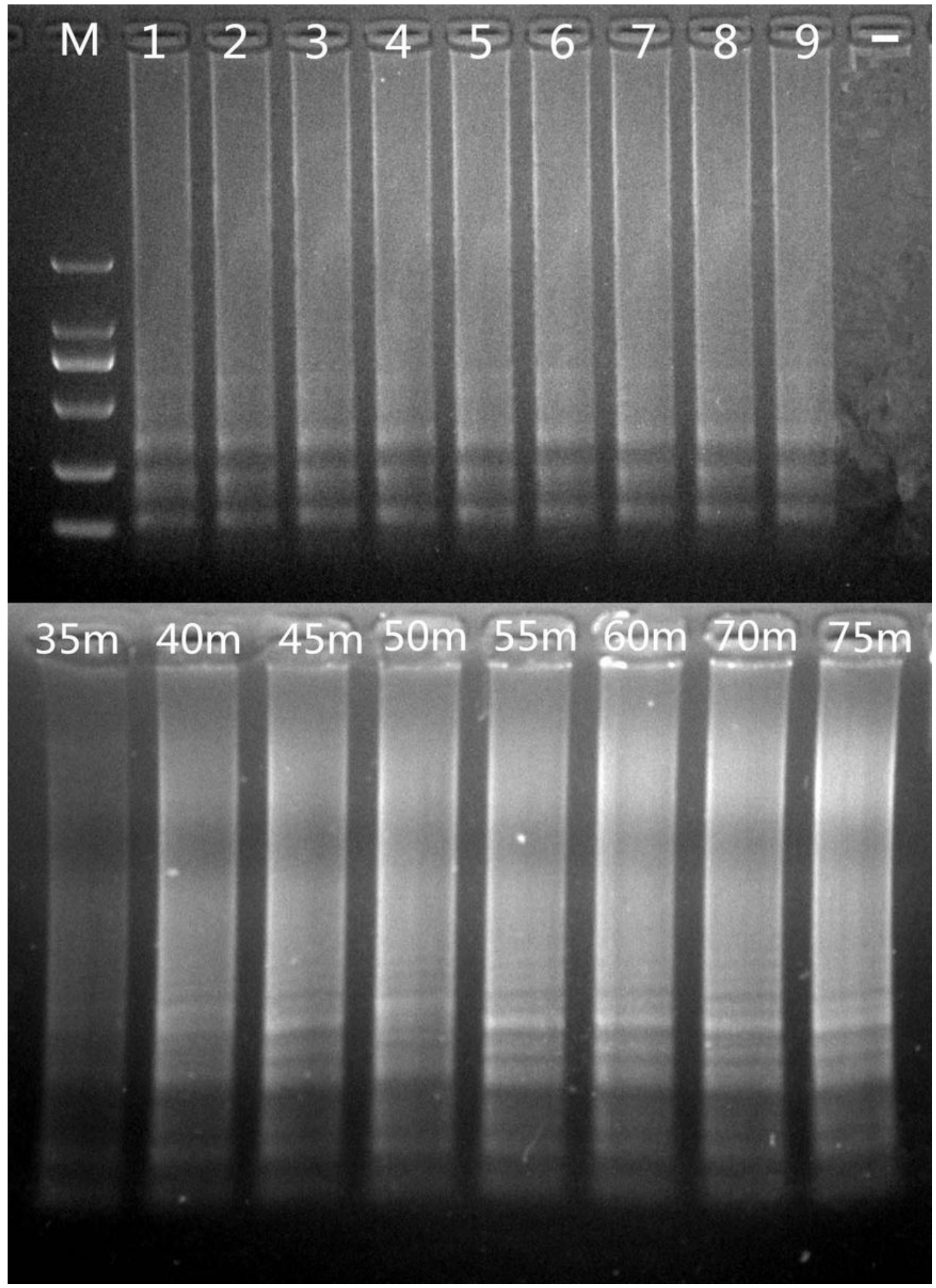

Figure 2

Experimental condition optimization $M \otimes D L 2000$ marker $1-9: 56 \sim 64^{\circ} \mathrm{C}-\varangle$ Negative control 35m-75m $\rrbracket 35-$ $75 \mathrm{~min}$ 


\section{$123 \mathrm{AB}$}

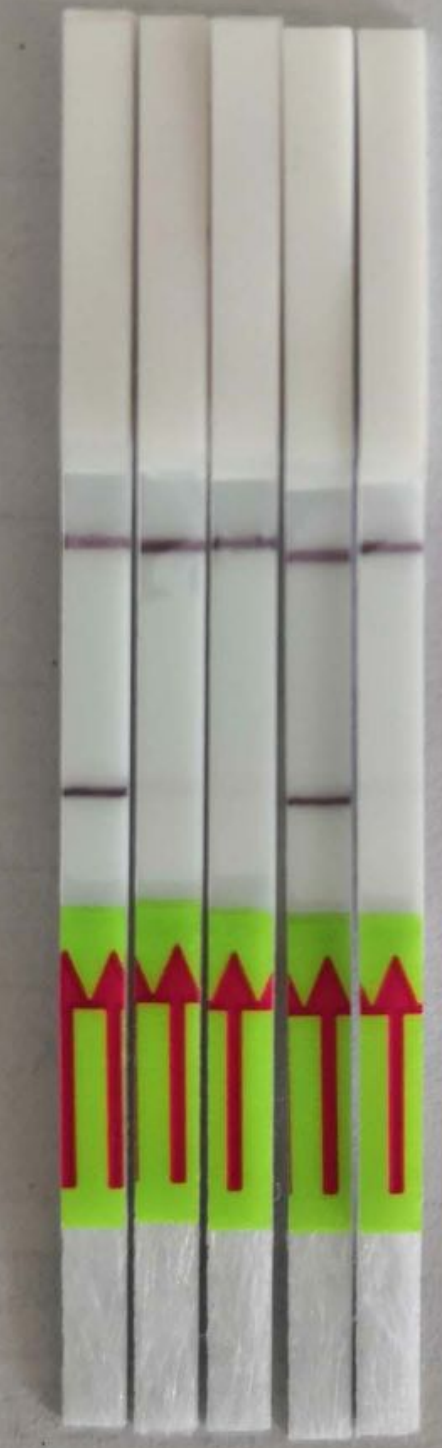

\section{Figure 3}

Screening hybridization probe 1هProbe 12 \Probe 2 3هProbe 3 A: Probe A B: Probe B 


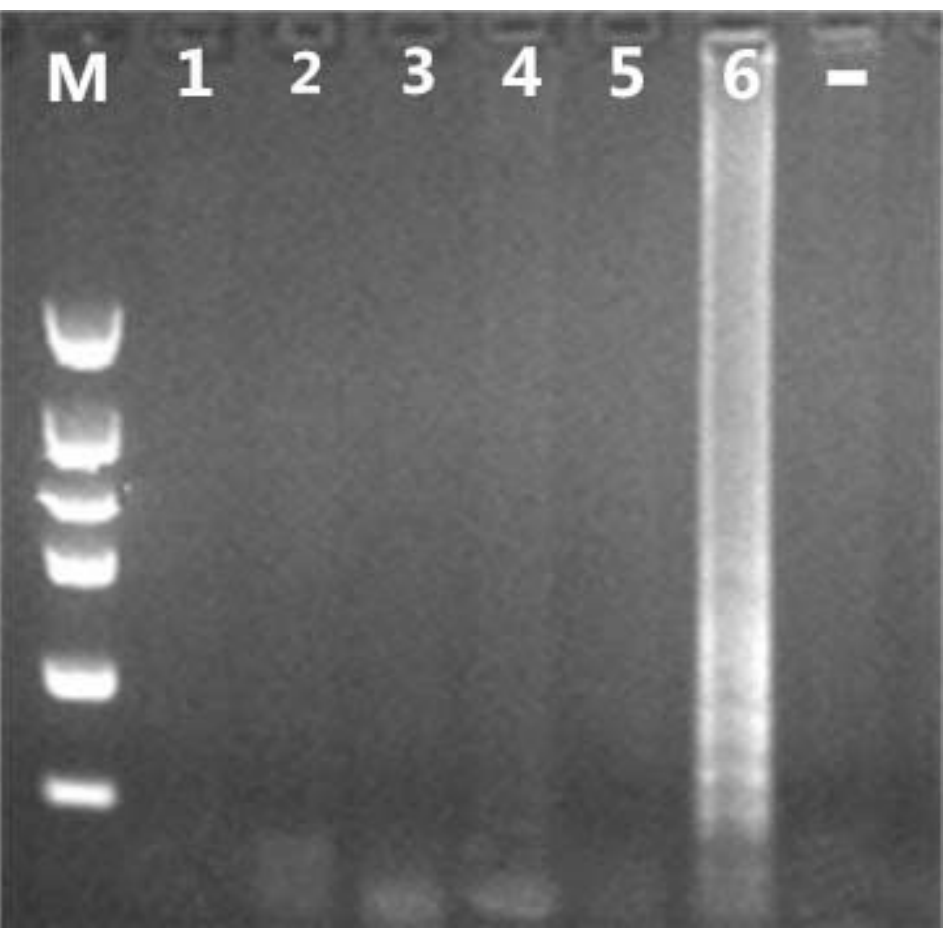

\section{Figure 4}

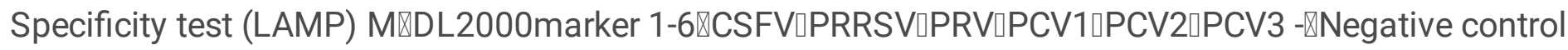

- PCV-3PCV-1 PCV-2 PRV CSFV PRRSV

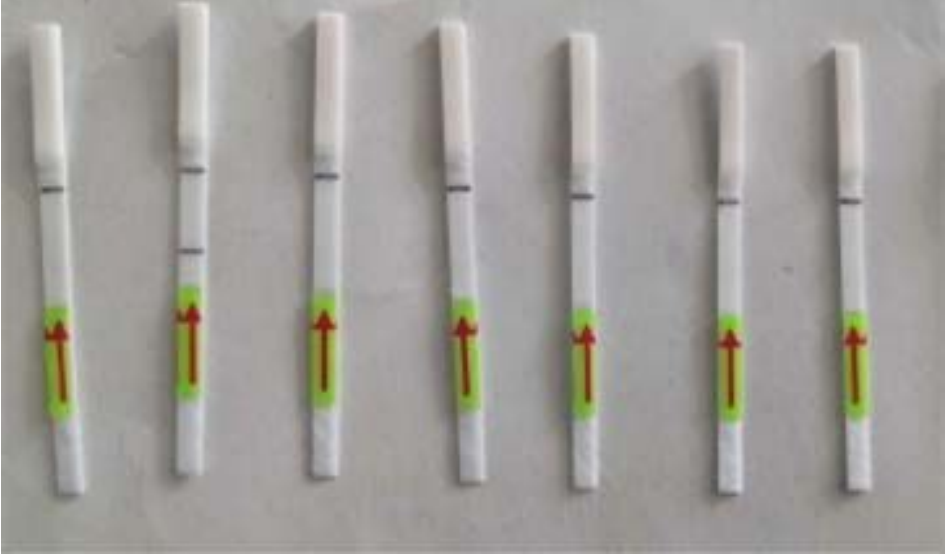

Figure 5

Specificity test (LAMP-LFD) M $₫ D L 2000$ marker 1-6هCSFV $\square P R R S V \square P R V \square P C V 1 \square P C V 2 \square P C V 3-\llbracket N e g a t i v e$ control 
500bp

250bp

\section{Figure 6}

Sensitivity test (conventional PCR method) M: DL2000 Marker -: Negative control 0: undiluted positive plasmid (200 ng/ $\mu \mathrm{L})$ 1-10:20 ng/ $\mu \mathrm{L}, 2 \mathrm{ng} / \mu \mathrm{L}, 0.2 \mathrm{ng} / \mu \mathrm{L}, 20 \mathrm{pg} / \mu \mathrm{L}, 2 \mathrm{pg} / \mu \mathrm{L}, 0.2 \mathrm{pg} / \mu \mathrm{L}, 20 \mathrm{fg} / \mu \mathrm{L}, 2 \mathrm{fg} / \mu \mathrm{L}$, $0.2 \mathrm{fg} / \mu \mathrm{L}, 20 \mathrm{ag} / \mu \mathrm{L}$ 


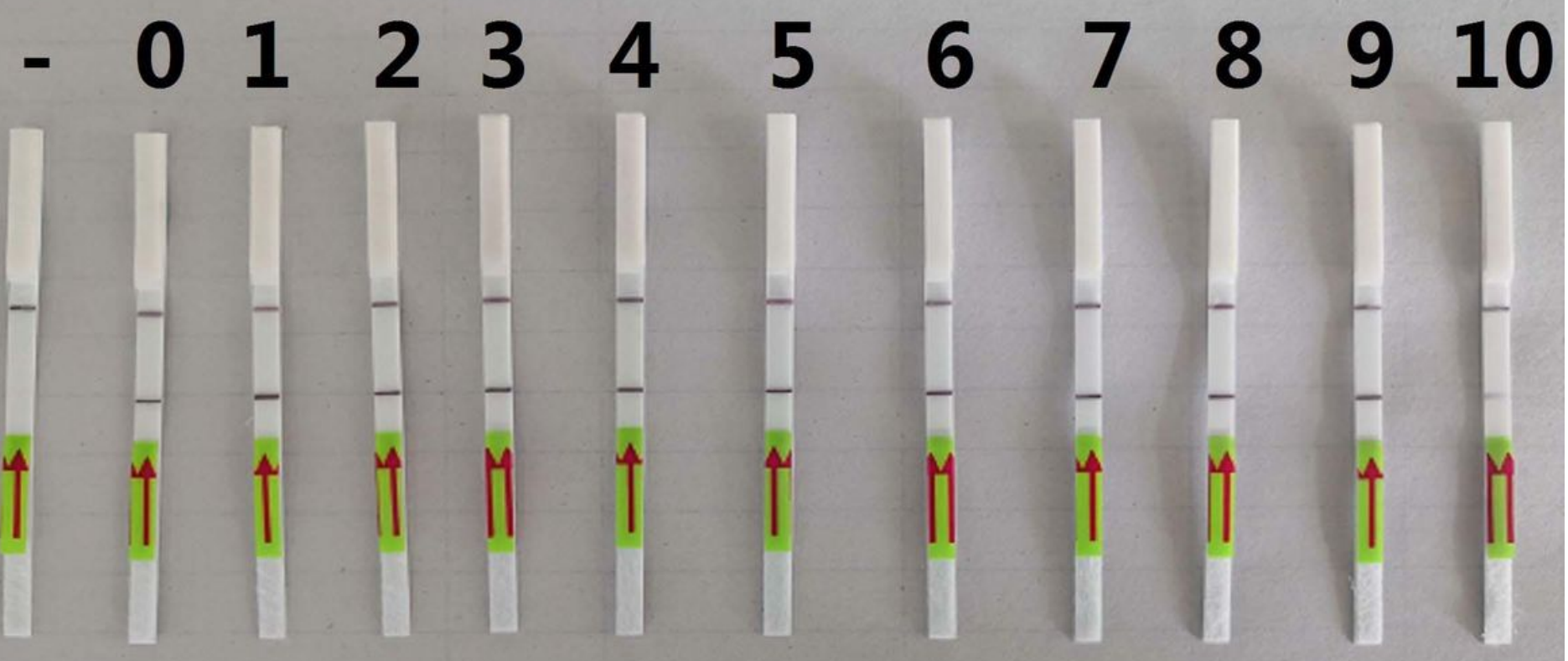

\section{Figure 7}

Sensitivity test (LAMP-LFD method) M: DL2000 Marker -: Negative control 0: undiluted positive plasmid (200 ng/ $\mu \mathrm{L}$ ) 1-10:20 ng/ $\mu \mathrm{L}, 2 \mathrm{ng} / \mu \mathrm{L}, 0.2 \mathrm{ng} / \mu \mathrm{L}, 20 \mathrm{pg} / \mu \mathrm{L}, 2 \mathrm{pg} / \mu \mathrm{L}, 0.2 \mathrm{pg} / \mu \mathrm{L}, 20 \mathrm{fg} / \mu \mathrm{L}, 2 \mathrm{fg} / \mu \mathrm{L}, 0.2 \mathrm{fg} /$ $\mu \mathrm{L}, 20 \mathrm{ag} / \mu \mathrm{L}$ 


\section{OAB}

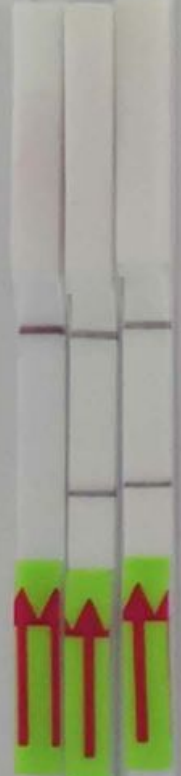

1

\section{OAB}

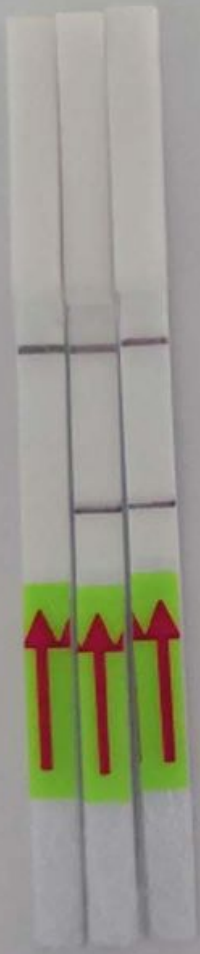

2
OAB

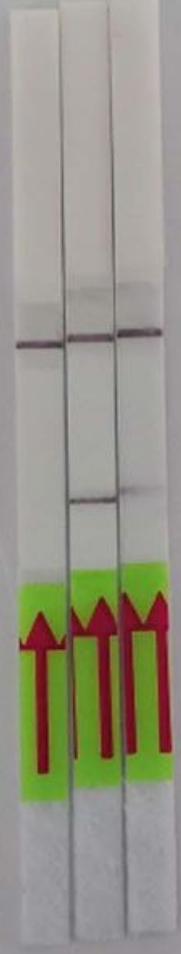

3
OAB

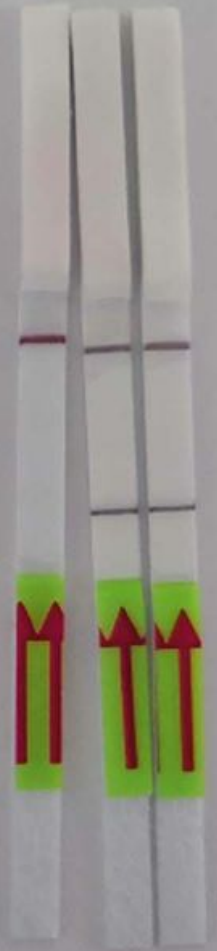

4
OAB

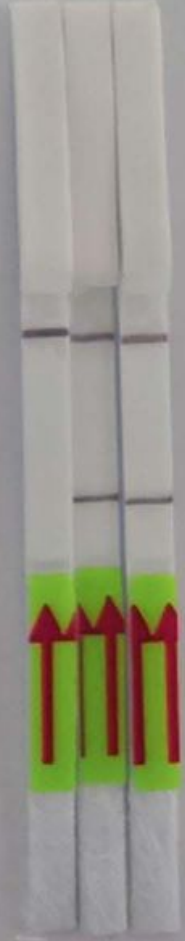

5

\section{Figure 8}

Repeatability test 0: Negative control A: undiluted positive plasmid (200 $\mathrm{ng} / \mu \mathrm{L}) \mathrm{B}$ : lowest concentration of plasmid in the sensitivity test $(0.2 \mathrm{fg} / \mu \mathrm{L})$ 1-5: 5 times LAMP-LFD test 


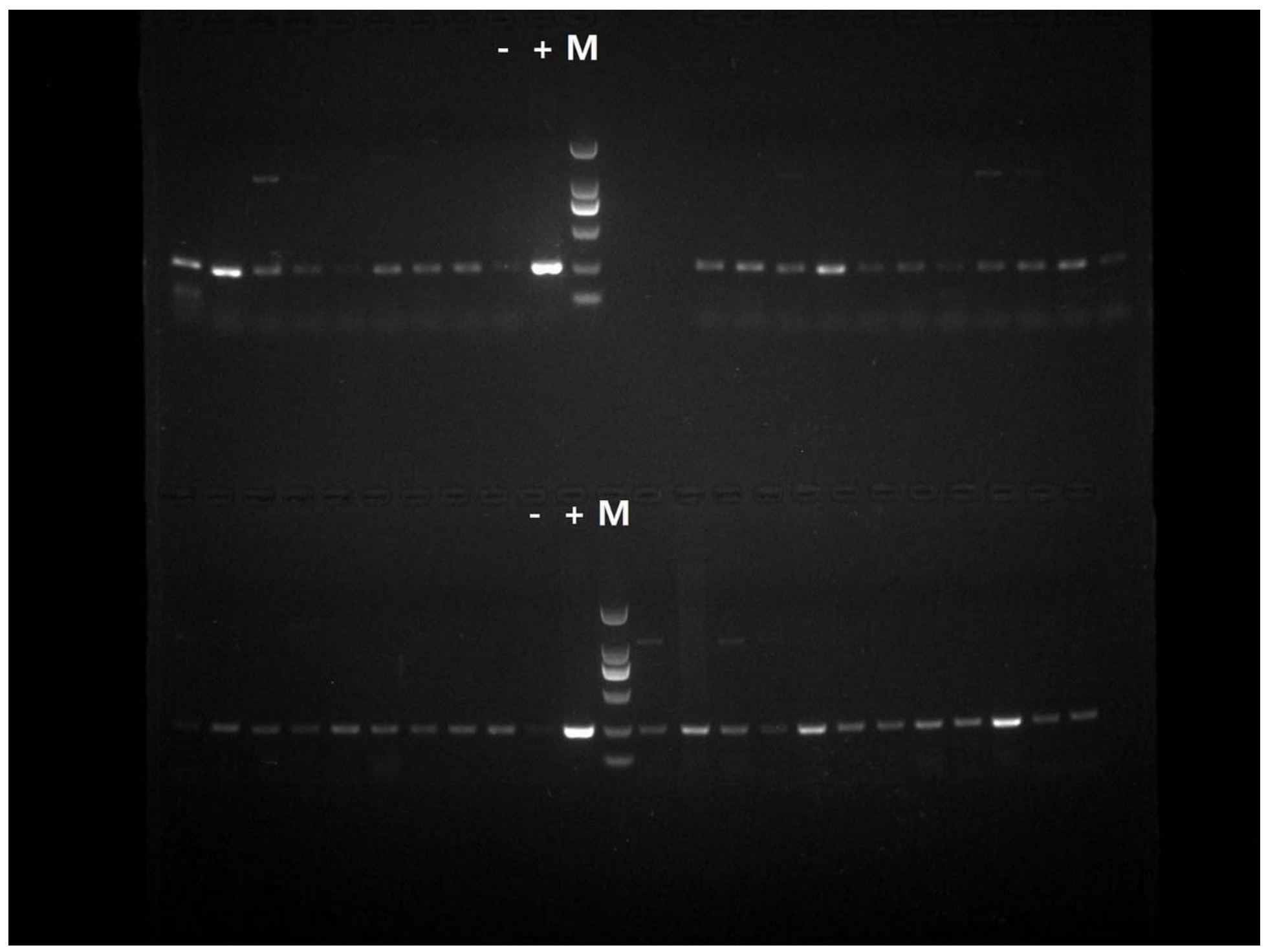

\section{Figure 9}

Results of partial sample (conventional PCR) M: DL2000marker -: Negative control +: positive control 


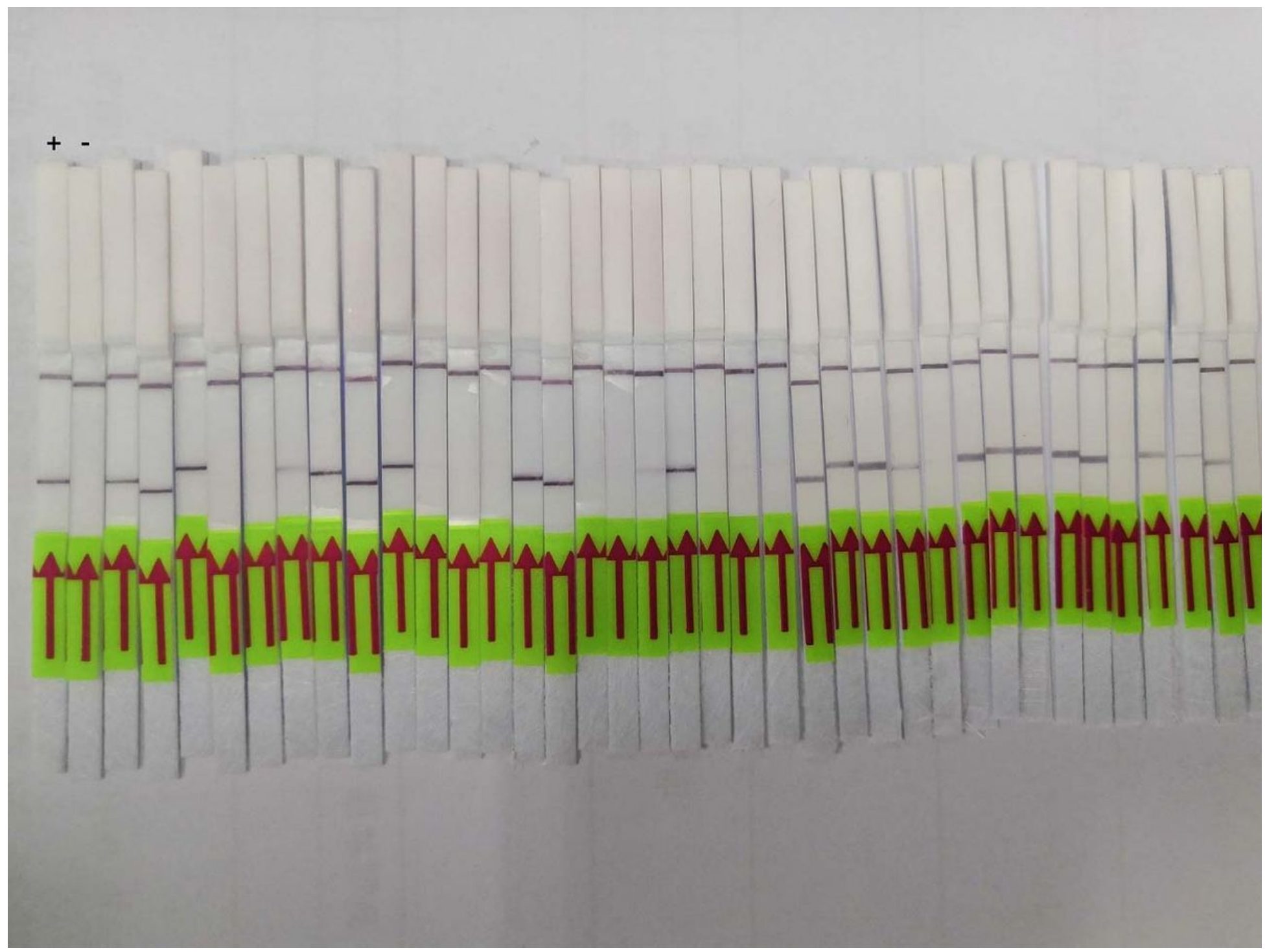

Figure 10

Results of partial sample (LAMP-LFD) M: DL2000marker -: Negative control +: positive control 